\title{
Relationship between Self-Perceived Age and Social Activity in Older Japanese Adults: The KAGUYA Study
}

\author{
Katsuhiko Takatori1,2, Daisuke Matsumoto ${ }^{1,2}$, Makoto Miyazaki ${ }^{3}$, Naomi Yamasaki ${ }^{4}$, \\ Jong-Seong Moon ${ }^{2,4}$ \\ ${ }^{1}$ Department of Physical Therapy, Faculty of Health Science, Kio University, Koryo, Kita-katsuragi-gun, Nara, Japan \\ ${ }^{2}$ Health Promotion Center, Kio University, Kita-katsuragi-gun, Nara, Japan \\ ${ }^{3}$ Center for Teaching, Learning and Technology, Kio University, Kita-katsuragi-gun, Nara, Japan \\ ${ }^{4}$ Department of Nurse, Faculty of Health Science, Kio University, Kita-katsuragi-gun, Nara, Japan \\ Email: k.takatori@kio.ac.jp
}

How to cite this paper: Takatori, K., Matsumoto, D., Miyazaki, M., Yamasaki, N. and Moon, J.-S. (2018) Relationship between Self-Perceived Age and Social Activity in Older Japanese Adults: The KAGUYA Study. Health, 10, 1459-1473.

https://doi.org/10.4236/health.2018.1011112

Received: October 1, 2018

Accepted: November 4, 2018

Published: November 7, 2018

Copyright $\odot 2018$ by authors and Scientific Research Publishing Inc. This work is licensed under the Creative Commons Attribution International License (CC BY 4.0).

http://creativecommons.org/licenses/by/4.0/

\begin{abstract}
Youthful feelings were shown to have a positive effect on the mental and physical conditions of the elderly. On the contrary, elderly people whose self-perceived age is older than their chronological age were shown to experience negative health outcomes. This study investigated whether the self-perceived age (younger or older) relative to chronological age promotes social activity. The baseline data acquired from 3094 older adults as part of the Keeping Active across Generations Uniting the Youth and the Aged study was used in the current study. The participants completed a questionnaire that solicits information pertaining to sociodemographic factors, felt age, self-rated health, physical activity, depression, disease burden, fall history, fear of falling, level of instrumental activities of daily living (IADLs) and social activity. The discrepancy between felt age and chronological age was defined as the self-perceived age. Self-perceived age was calculated by subtracting the participant's chronological age from the felt age, and the score for the difference was divided by the chronological age. The Japan Science and Technology Agency Index of Competence was used to evaluate social activity. Regression analysis results revealed that a younger self-perceived age was associated with higher social activity after controlling for sociodemographic factors, self-rated health, depression, physical activity, disease burden, level of IADL, fall history and fear of falling. Fall history was significantly associated with higher social activity in young-old participants $(65-74$ years) $(\mathrm{p}=$ 0.014 ) but not in old-old participants ( $>75$ years). This study suggests that a younger self-perceived age in older adults would lead to a high-level living function. This finding may provide useful information for interventions
\end{abstract}


aimed at the promotion of health and/or the prevention of frailty.

\section{Keywords}

Self-Perceived Age, Older Adults, Social Activity

\section{Introduction}

Self-perceived age refers to subjective age compared with chronological age. Previous studies have used terms such as "subjective age" [1] [2] [3] [4], "felt age" [5] and "cognitive age" [6] to describe the self-perceived age. Several studies have found that the discrepancy between perceived age and chronological age is associated with various health-related outcomes in elderly people. For example, people with younger subjective ages were shown to have better physical functions (physical activity, walking speed, grip strength and standing balance) [4] [6] [7], better cognitive function (memory and executive functions) [4] [6], greater feeling of well-being [8], fewer depressive symptoms [9], greater life satisfaction [1] and a lower degree of systemic inflammation than people with older subjective ages [10]. On the contrary, older subjective age was shown to be associated with adverse health outcomes (e.g., cognitive decline) and sedentary lifestyle [4]. In a longitudinal study that investigated the relationship between subjective age and risk of death, individuals with older subjective ages experienced higher mortality rates than individuals with younger subjective ages [11]. These findings suggest that subjective age is a convenient surrogate index that can be used to evaluate health, in addition to self-rated health. Factors that affect subjective age have been widely investigated. High physical activity level [12], low psychological distress [13], good self-rated health, personality traits of extraversion and openness [2], good mental health and high personal mastery [14] were shown to be associated with younger subjective age.

However, the relationship between social activities and subjective age is not well characterised even though the relationship of physical and psychological factors with subjective age has been widely reported. The only study that prospectively investigated the covariates of subjective age showed that a decrease in loneliness was associated with a younger subjective age, whereas an increase in depressive symptoms was associated with older subjective age [15]. With respect to the relationship between subjective age and social activity in the elderly, including participation in community activities and social roles that rank higher than instrumental activities of daily living (IADLs), one study evaluated the number of social contacts such as conversations and telephone calls. However, according to the hierarchical model of competence of [16], the relationship between high-level living function and self-perceived age has not been sufficiently investigated. We hypothesised that a lower subjective age is a factor that promotes social activity and that there is an interaction between subjective and chronological age. 
The objective of this study was to clarify the relationship between self-perceived age and social activity in daily life by using baseline data from currently ongoing large-scale cohort studies and to provide useful data for the prevention of frailty in the elderly.

\section{Methods}

\subsection{Participants}

Participants were drawn from the Keeping Active across Generations Uniting the Youth and the Aged (KAGUYA) study. The KAGUYA study is a nationally representative five-year longitudinal study supported by the Ministry of Education, Culture, Sports, Science and Technology (Private University Strategic Research Base Formation Project, 2015-2020). The aim of the KAGUYA study is to identify the factors that affect a long and healthy life span by conducting a questionnaire survey of all elderly residents of Koryo Town, Nara Prefecture, Japan. The questionnaire ascertained if the rates of certification for nursing care decreased and if a healthy life expectancy extended to those who received interventions. It also aimed to determine the effects of specific health promotion interventions (care prevention classes, an Alzheimer's café wherein affected individuals and families may gather, etc.). The questionnaire was mailed to all people in Koryo Town $(n=8004)$ who are aged $\geq 65$ years. Among the 3871 people who returned the questionnaire (response rate: $48.3 \%$ ), data pertaining to 3094 individuals were included in the analysis (final inclusion rate: $38.7 \%$ ). Those who were certified as eligible for nursing care under long-term care insurance were excluded from the analysis. Fifty-two percent of the participants were women, and the average age of the participants was 72.9 years $(\mathrm{SD}=6.2$; range $65-98$ years). In addition to sociodemographic characteristics, the questionnaire included aspects of felt age, physical activity (exercise habit), IADL, social activity (high-level functional capacity), disease burden, depressive symptoms and history of falls.

The protocol for this study and the study procedures were approved by the ethics committee at the Kio University in Japan (Approval No. H27-34). All participants signed a consent form that was included in the survey questionnaire.

This study was registered to UMIN-CTR (UMIN 000019803) on 2 December 2015.

\subsection{Measures}

\subsubsection{Self-Perceived Age}

In this study, as described by a previous study [11], we defined the age that was felt subjectively by participants as "felt age" and defined the magnitude of the discrepancy with chronological age as "self-perceived age". In a previous study [3], felt age was assessed by asking participants to specify how old they felt (in years). In another study [17], self-perceived age was defined as the discrepancy between the chronological age and the felt age (calculated as felt age minus 
chronological age). The difference was then divided by the chronological age. Therefore, a lower value indicated a younger self-perceived age, and a higher value indicated an older self-perceived age. In this analysis, we excluded 20 participants who provided extreme responses (three standard deviations above or below the mean) as outliers.

\subsubsection{Social Activity (High-Level Functional Capacity)}

Functional capacity is a general term for the various physical and mental functions that are necessary for older people to lead their daily lives. The hierarchical model of competence by Lawton [16] categorises functional capacity into the following seven conceptual sublevels: 1) life maintenance, 2) functional health, 3) perception-cognition, 4) physical self-maintenance, 5) instrumental self-maintenance, 6) effectance (intellectual activity) and 7) social role. The fourth sublevel in this model is consistent with the degree of independence in the activity of daily living (ADL), and the fifth to the seventh sublevels show high-level functional capacity. Inthe process of the development of frailty in the elderly, functional degradation begins with the highest area of competence [18]. On the basis of Lawton's model, Koyano et al. [19] developed the Tokyo Metropolitan Institute of Gerontology Index of Competence (TMIG-IC) to measure three high-level functional capacities. High-level functional capacity is positioned above the basic ADLs and corresponds to the fifth, sixth and seventh sublevels of Lawton's hierarchical model. Furthermore, to account for changes in the living environment of older people in recent years, Iwasa et al. [20] [21] expanded the concept of the 3 TMIG-IC categories and developed the 16-item Japan Science and Technology Agency Index of Competence (JST-IC). This index is positioned higher than TMIG-IC and measures a higher level of daily function that includes newly added components such as social participation and life management. JST-IC can measure the "competences required for older individuals living alone to become independent and lead an active daily life" within the living environment of the modern active older citizen; furthermore, it has properties that require higher functioning to execute compared with the ADL and IADL [19] [22]. JST-IC is an evaluation index whose reliability and validity were verified in a previous research [21].

In this study, we focused on life management and social participation and these were defined as "social activity" under competence for higher living functions (as described above), and JST-IC was used as a quantitative evaluation index. The domains of JST-IC include the following: 1) device usage (adapting to continuous developments in electrical appliances and ICT devices), 2) information gathering (attitude and behaviour related to the information necessary for one's safety, health and everyday life and to decision-making based on this information), 3) productive activity (performing productive activities that are free, such as caring for others, producing goods, providing services and performing economic activities) and 4) social participation (focuses on activities for regional promotion). Table 1 shows the specific question items. 
Table 1. The English version of the Japan Science and Technology Agency Index of Competence (JST-IC).20).

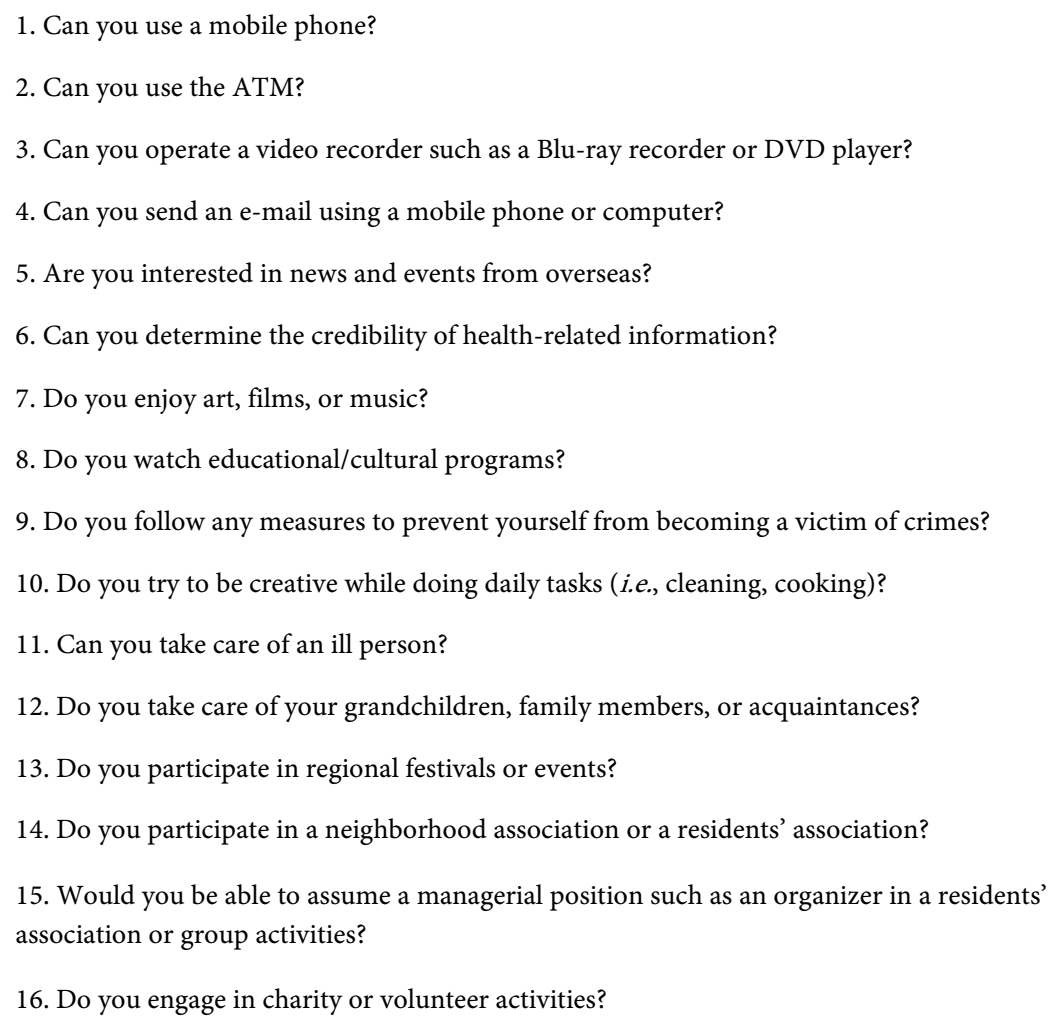

The above items were translated from the Japanese version of the scale. The English version of this scale has not yet been validated.

\subsubsection{Covariates}

The sociodemographic covariates included age (in years), sex (coded as " 1 " for men and " 0 " for women), living alone or not and educational history (in years). The health-related covariates included disease burden, self-rated health, depressive symptoms, fear of falling and history of falls. Physical activity and level of IADL were also included as behavioural covariates.

\subsubsection{Physical Activity}

Physical activity was evaluated by the presence or absence of an exercise habit. According to the definition used in the National Health and Nutrition Survey [23], we defined exercise habit as engaging in continuous exercises for more than 30 minutes twice a week for more than 1 year. Movements during daily activities, such as walking while shopping, were not included in this definition.

\subsubsection{IADLs}

We used the items related to the IADL from TMIG-IC [19] to assess the IADLs level. TMIG-IC is a widely used standard instrument in Japan and comprises 13 multidimensional items. Its reliability and validity were tested [22]. Items 1 - 5 solicit information on the IADLs as follows: Item 1: Can you use public transportation (bus or train) by yourself? Item 2: Are you able to shop for daily ne- 
cessities? Item 3: Are you able to prepare meals by yourself? Item 4: Are you able to pay bills? Item 5: Can you handle your own banking? The response to each item is "yes" (able to do) or "no" (unable to do), and each "yes" or "no" answer is scored as "1" or "0", respectively.

\subsubsection{Self-Rated Health}

Self-rated health was assessed using a single item that asked participants to report whether their health was excellent, very good, good, fair or poor. We reversed the original scale such that excellent health received the highest score (five points) and poor health received the lowest score (one point).

\subsubsection{Depression Symptoms}

The Geriatric Depression Scale (GDS) is a 30-item self-reported assessment tool that was designed specifically to identify depression in the elderly. In this study, we used the five-item GDS [24] [25], which is a validated simplified version of the GDS. Participants responded with either "Yes" or "No" to each question. Each "Yes" was scored as "1", and each "No" was scored as "0". Depressive symptoms were suggested by an answer of "Yes" to two or more of the five items.

\subsubsection{Disease Burden}

The sum of the diagnosed conditions (i.e., hypertension, diabetes, heart disease, osteoporosis, dyslipidemia, stroke or arthritis) was computed to obtain the measure of disease burden.

\subsubsection{History of Falls and Fear of Falling}

The history of falls was evaluated on the basis of the history of falls over the past year. Fear of falling was evaluated by the presence or absence of anxiety about a future fall.

This template, created in MS Word 2007, provides authors with most of the formatting specifications needed for preparing electronic versions of their papers. All standard paper components have been specified for three reasons: 1) ease of use when formatting individual papers, 2) automatic compliance to electronic requirements that facilitate the concurrent or later production of electronic products, and 3) conformity of style throughout a journal paper. Margins, column widths, line spacing, and type styles are built-in; examples of the type styles are provided throughout this document and are identified in italic type, within parentheses, following the example. Some components, such as multi-leveled equations, graphics, and tables are not prescribed, although the various table text styles are provided. The formatter will needto create these components, incorporating the applicable criteria that follow.

\subsection{Data Analysis}

Paired $t$-test was used to compare a participant's chronological age with felt age. We also compared the felt age and self-perceived age by sex. Multiple regression 
analysis was conducted with JST-IC score as the dependent variable and with all study factors as independent variables. To examine the independent contribution of self-perceived age to social activity, they were tested in separate models. In the first model, age, sex, living alone or not, educational level, disease burden and self-perceived age were entered as independent variables. In the second model, in addition to the independent variables in the first model, items related to self-rated health and depressive symptoms were entered as independent variables. In the third model, in addition to those in the second model, independent variables related to falls were entered (i.e., fear of falling and history of falls). In the final model, in addition to those in the third model, the IADL level and physical activity were included as independent variables. To investigate the influence of age, participants were classified as young-old participants (age range, 65 - 75 years) and as old-old participants (>75 years), and a regression model was created by the same procedure. Collinearity diagnostics did not indicate any sign of multicollinearity. All statistical analyses were conducted using SPSS version 21.0 (IBM, Chicago, IL, USA). The level of significance was set at $\mathrm{p}<0.05$.

\section{Results}

Table 2 shows the characteristics of the participants. The average chronological age was 72.9 years ( $\mathrm{SD}=6.2$, range: 65 - 98 years), and the average felt age was 66.7 years ( $\mathrm{SD}=7.5$, range: $48-91)$. There was no sex difference in felt age, and felt age was significantly younger than chronological age $(\mathrm{p}<0.01)$. The average self-perceived age was $-0.10(\mathrm{SD}=0.08)$. The mean TMIG-IC (IADL) and JST-IC scores were $4.69(\mathrm{SD}=0.76)$ and $10.15(\mathrm{SD}=3.44)$, respectively. These results are similar to the average values from participants aged 65 - 74 years reported from a previous study [26] of older adults in the general population.

The results of multiple regression analysis after controlling for sociodemographic variables (Table 3 ) showed that self-perceived age was negatively related to higher social activity in daily living. This result suggested that individuals who feel younger than their chronological age have higher social activity. In the second model, lower self-perceived age, lower depressive status and higher self-rated health was associated with higher social activity. In the third model, fear of falling was also associated with higher social activity. In the final model, self-perceived age showed a significant association with higher social activity even after controlling for other factors; the coefficient for self-perceived age decreased from the first model to the final model but remained significant. The explained variance (adjusted $\mathrm{R}^{2}$ ) increased significantly when physical activity and TIMG-IC (IADL) were added to the model $(\mathrm{p}<0.01)$. No significant association was observed between disease burden or a history of fall and social activity.

The relationship between self-perceived age and social activity was also significant in all models in the subanalysis performed by disaggregating participants into young-old (65 - 74 years) and old-old (>75 years) subgroups (Table 4 and Table 5). In both groups, the explained variance increased significantly in 
the second model, which incorporated self-rated health and depressive state (in addition to the sociodemographic variables), and in the final model, which additionally incorporated physical activity and TIMG-IC (IADL). A history of falls was negatively associated with social activity in the old-old participants, but not in the young-old participants. On the contrary, fear of fall showed a significant positive association in the young-old subgroup, but not in the old-old subgroup.

Furthermore, a comparison of self-perceived age based on physical activity showed that subjects who engaged in physical activity had significantly lower self-perceived age than subjects who did not engage in physical activity $(\mathrm{p}<$ 0.01) (Figure 1). This result suggested that individuals with an exercise habit tend to feel younger than their chronological age.

Table 2. Participants characteristics $(\mathrm{N}=3074)$.

\begin{tabular}{|c|c|c|}
\hline Variables & Mean/N (\%) & SD \\
\hline Age (65 - 98) & 72.9 & 6.2 \\
\hline Sex (female) & $1598(52 \%)$ & - \\
\hline Living alone & $261(8.5 \%)$ & - \\
\hline \multicolumn{3}{|l|}{ Education } \\
\hline$<6$ Years & $18(0.6 \%)$ & - \\
\hline $6 \leq 9$ Years & $700(22.8 \%)$ & - \\
\hline $10 \leq 12$ Years & $1420(46.2 \%)$ & - \\
\hline$\geq 13$ Years & $919(29.9 \%)$ & - \\
\hline Self-rated Health & 3.51 & 1.00 \\
\hline Felt age & 66.73 & 7.50 \\
\hline Self-perceived age ${ }^{a}$ & -0.10 & 0.08 \\
\hline Exercise habit & $1238(40.3 \%)$ & - \\
\hline Disease Burden & 1.16 & 0.94 \\
\hline TMIG-IC (IADL) & 4.69 & 0.76 \\
\hline JST-IC & 10.15 & 3.44 \\
\hline GDS-5 & 1.56 & 0.96 \\
\hline Fall history (past 1y) & $451(14.7 \%)$ & - \\
\hline Fear of falling & $1475(48 \%)$ & - \\
\hline
\end{tabular}

Notes: TMIG-IC: Tokyo Metropolitan Institute of Gerontology Index of Competence; JST-IC: Japan Science and Technology Agency Index of Competence; GDS-5: the five-item geriatric depression scale. aSelf-perceived age $=($ Felt age-Chronological age $) /$ Chronological age. Lower value represent younger self-perceived age. Twenty participants who were outliners on subjective age were excluded from the analysis. 
Table 3. Multivariate regression analysis predicting JST-IC score (All participants).

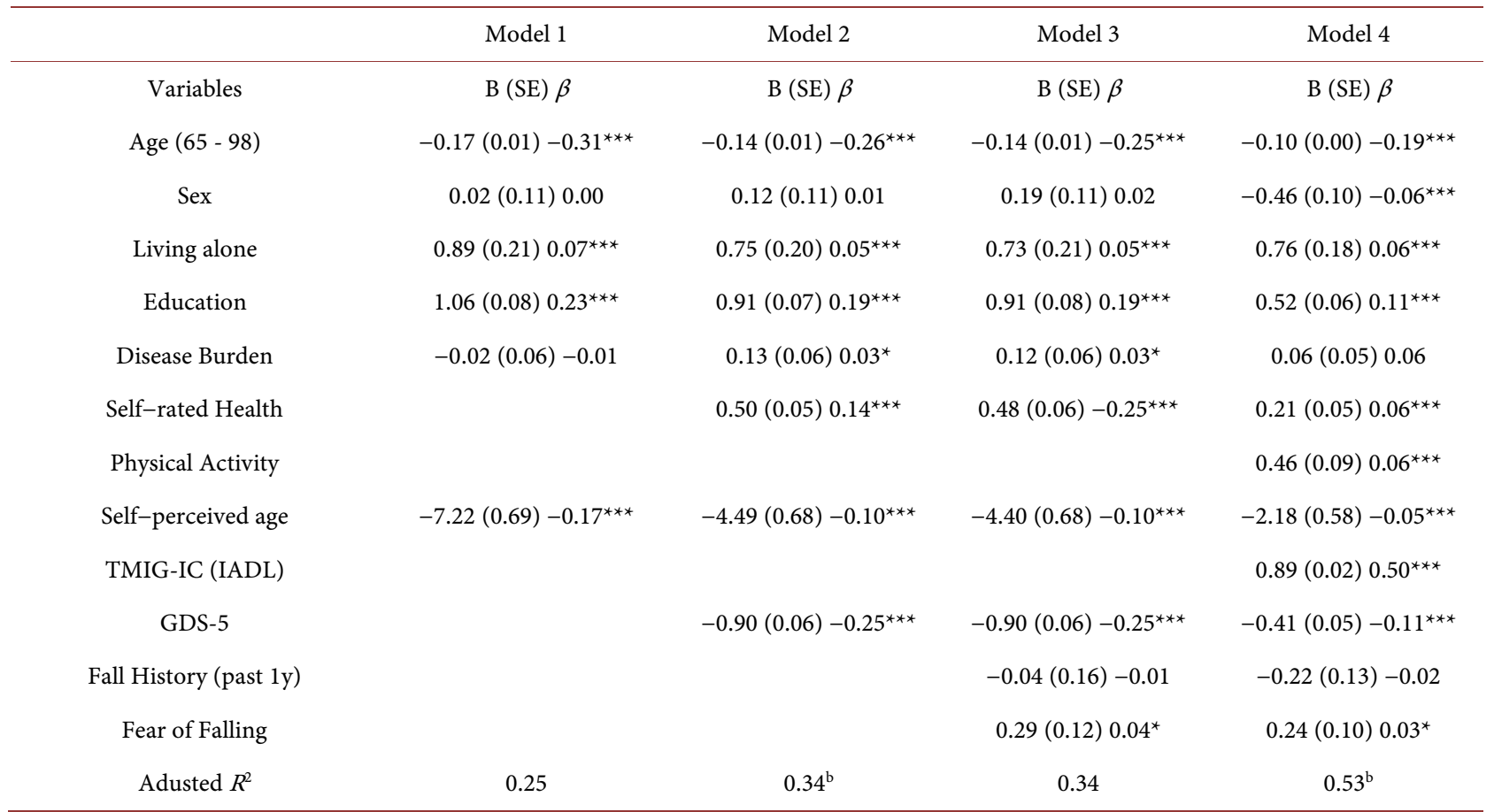

Notes: TMIG-IC: Tokyo Metropolitan Institute of Gerontology Index of Competence; JST-IC: Japan Science and Technology Agency Index of Competence; GDS-5: the five-item geriatric depression scale.B unstandardized regression coefficient, SE standard error, $\beta$ standardized regression coefficient. A Self-perceived age $=($ Felt age-Chronological age $) /$ Chronological age. $\mathrm{b}$ These data show a significant increase in explained variance $(\Delta \mathrm{F}$ with $\mathrm{p}<0.001) .{ }^{\star} \mathrm{p}<$ $0.05 ;{ }^{* *} \mathrm{p}<0.01,{ }^{* *} \mathrm{p}<0.001$

Table 4. Multivariate regression analysis predicting JST-IC score (Young-old participants).

\begin{tabular}{|c|c|c|c|c|}
\hline & Model 1 & Model 2 & Model 3 & Model 4 \\
\hline Variables & $\mathrm{B}(\mathrm{SE}) \beta$ & $\mathrm{B}(\mathrm{SE}) \beta$ & $\mathrm{B}(\mathrm{SE}) \beta$ & $\mathrm{B}(\mathrm{SE}) \beta$ \\
\hline Sex & $0.60(0.13) 0.10^{\star * *}$ & $0.70(0.12) 0.11^{\star * *}$ & $0.76(0.13) 0.12^{\star * *}$ & $-0.16(0.11)-0.02$ \\
\hline Living alone & $1.21(0.25) 0.10^{\star * *}$ & $1.16(0.25) 0.10^{* * *}$ & $1.12(0.25) 0.09^{\star * *}$ & $1.03(0.21) 0.08^{\star * *}$ \\
\hline Education & $1.07(0.09) 0.25^{\star * *}$ & $0.95(0.09) 0.22^{\star * *}$ & $0.95(0.09) 0.22^{* * *}$ & $0.52(0.08) 0.12^{\star * *}$ \\
\hline Disease Burden & $-0.03(0.07)-0.01$ & $0.16(0.07) 0.05^{\star}$ & $0.16(0.07) 0.05^{\star}$ & $0.14(0.06) 0.04^{*}$ \\
\hline Self-rated Health & & $0.53(0.06) 0.17^{\star * *}$ & $0.50(0.07) 0.16^{* * *}$ & $0.25(0.06) 0.08^{\star * *}$ \\
\hline Physical Activity & & & & $0.29(0.11) 0.04^{\star}$ \\
\hline TMIG-IC (IADL) & & & & $0.94(0.03) 0.53^{* * *}$ \\
\hline GDS-5 & & $-0.72(0.07)-0.21^{\star \star *}$ & $-0.70(0.07)-0.20^{\star * *}$ & $-0.26(0.06)-0.07^{\star \star \star}$ \\
\hline Fall History (past 1y) & & & $0.17(0.20) 0.01$ & $0.04(0.17) 0.00$ \\
\hline Fear of Falling & & & $0.38(0.14) 0.06^{* *}$ & $0.24(0.12) 0.04^{*}$ \\
\hline Adusted $R^{2}$ & 0.12 & $0.21^{\mathrm{b}}$ & 0.22 & $0.44^{\mathrm{b}}$ \\
\hline
\end{tabular}

Notes: TMIG-IC: Tokyo Metropolitan Institute of Gerontology Index of Competence;JST-IC: Japan Science and Technology Agency Index of Competence; GDS-5: the five-item geriatric depression scale. B unstandardized regression coefficient, SE standard error, $\beta$ standardized regression coefficient. aSelf-perceived age $=($ Felt age-Chronological age $) /$ Chronological age. b These data show a significant increase in explained variance $(\Delta \mathrm{F}$ with $\mathrm{p}<0.001) .{ }^{\star} \mathrm{p}$ $<0.05 ;{ }^{* *} \mathrm{p}<0.01,{ }^{* * *} \mathrm{p}<0.001$. 
Table 5. Multivariate regression analysis predicting JST-IC score (Old-old participants).

\begin{tabular}{|c|c|c|c|c|}
\hline & Model 1 & Model 2 & Model 3 & Model 4 \\
\hline Variables & $\mathrm{B}(\mathrm{SE}) \beta$ & $\mathrm{B}(\mathrm{SE}) \beta$ & $\mathrm{B}(\mathrm{SE}) \beta$ & $\mathrm{B}(\mathrm{SE}) \beta$ \\
\hline Age (65 - 98) & $-0.30(0.02)-0.35^{\star * *}$ & $-0.23(0.02)-0.27^{\star * *}$ & $-0.23(0.02)-0.27^{\star * *}$ & $-0.13(0.02)-0.15^{\star \star \star}$ \\
\hline Sex & $-1.06(0.21)-0.14^{\star * *}$ & $-1.06(0.20)-0.14^{\star * *}$ & $-0.98(0.21)-0.13^{\star * *}$ & $-1.15(0.19)-0.15^{\star * *}$ \\
\hline Living alone & $0.25(0.36) 0.02$ & $-0.02(0.34)-0.00$ & $-0.03(0.35)-0.00$ & $0.20(0.31) 0.01$ \\
\hline Education & $1.16(0.13) 0.24^{* * *}$ & $0.91(0.13) 0.18^{\star * *}$ & $0.95(0.13) 0.19^{* * *}$ & $0.58(0.12) 0.12^{* * *}$ \\
\hline Disease Burden & $0.01(0.10) 0.00$ & $0.09(0.10) 0.02$ & $0.08(0.10) 0.02$ & $-0.03(0.09)-0.01$ \\
\hline Self-rated Health & & $0.48(0.10) 0.13^{\star * *}$ & $0.47(0.11) 0.12^{\star * *}$ & $0.17(0.10) 0.04$ \\
\hline Physical Activity & & & & $0.66(0.19) 0.08^{* *}$ \\
\hline Self-perceived age $\mathrm{a}^{\mathrm{a}}$ & $-10.9(1.51)-0.20^{\star * *}$ & $-7.21(1.47)-0.13^{\star * *}$ & $-7.16(1.51)-0.13^{\star * *}$ & $-3.76(1.32)-0.07^{\star *}$ \\
\hline TMIG-IC (IADL) & & & & $0.77(0.04) 0.47^{\star * *}$ \\
\hline GDS-5 & & $-1.00(0.10)-0.29^{\star * *}$ & $-1.03(0.10)-0.30^{\star * \star}$ & $-0.62(0.09)-0.18^{\star * *}$ \\
\hline Fall History (past 1y) & & & $-0.34(0.25)-0.03$ & $-0.56(0.22)-0.06^{*}$ \\
\hline Fear of Falling & & & $0.07(0.23) 0.01$ & $0.19(0.20) 0.02$ \\
\hline Adusted $R^{2}$ & 0.29 & $0.40^{\mathrm{b}}$ & 0.40 & $0.57^{\mathrm{b}}$ \\
\hline
\end{tabular}

Notes: TMIG-IC: Tokyo Metropolitan Institute of Gerontology Index of Competence; JST-IC: Japan Science and Technology Agency Index of Competence; GDS-5: the five-item geriatric depression scale. B unstandardized regression coefficient, SE standard error, $\beta$ standardized regression coefficient aSelf-perceived age $=($ Felt age-Chronological age $) /$ Chronological age. $\mathrm{b}$ These data show a significant increase in explained variance $(\Delta \mathrm{F}$ with $\mathrm{p}<0.001) .{ }^{\star} \mathrm{p}$ $<0.05 ;{ }^{* *} \mathrm{p}<0.01,{ }^{* * *} \mathrm{p}<0.001$.

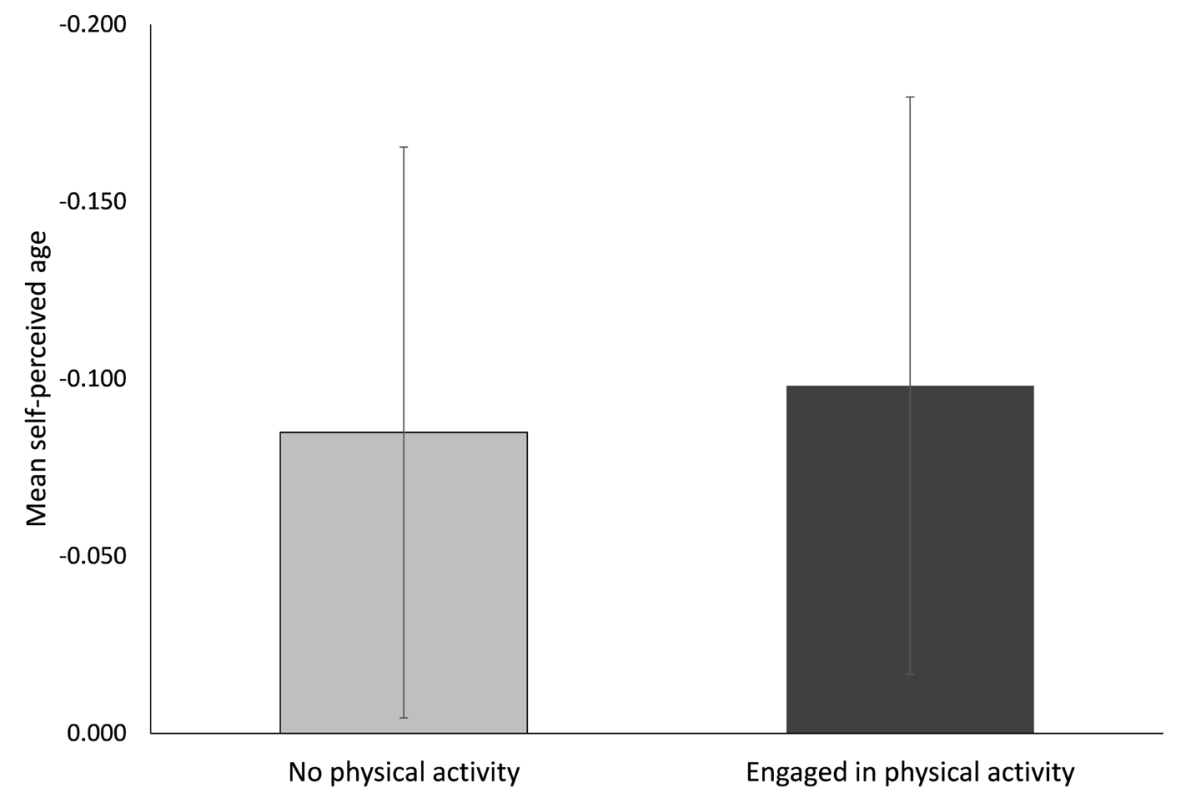

Figure 1. Comparison of self-perceived age between presence or absence of physical activity. Notes: Values: Mean, Error bar: standard deviation ${ }^{\star *} \mathrm{p}<0.01$.

\section{Discussion}

To understand the concept of old age, it is necessary to distinguish between biological age, functional age and chronological age. Chronological age (number of 
years elapsed since birth) is the most common indicator used to define old age. Biological age (also referred to as primary ageing) is the body's actual biological age compared with the standard markers of biological age in the general population. It refers to pathophysiological processes that eventually lead to loss of adaptability, onset of disease, physical impairment, functional limitations, disability and eventual death [27]. Functional age can be defined as the state of health of an individual compared with that of other people of the same age and gender. Older adults have a lower capacity to cope with diseases and adapt to environmental changes: they tend to tire more easily, are slower and exhibit less mobility and agility. Furthermore, their perceptive skills, mental agility and memory are lower. An increase in subjective age presents a risk of lowered living function, and a relationship of subjective age with the risk of dementia and heart disease has been reported [11]. All of these factors condition the functional capacity [28], which is a concept that reflects an older adult's ability to perform physical activities in daily life with relative ease [29]. Even in healthy adults, each component of functional capacity decreases with advancing age, thus negatively affecting the quality of life [30]. Social and cultural values from the environment also directly affect the ageing process. In a study that tracked community elderly groups using full TMIG-IC scores, "intellectual activity" and "social role" were found susceptible to disability a few years prior to disability in IADLs [18].

In the current study, felt age was significantly lower than the chronological age; this result is consistent with those of previous studies [9] [31]. We calculated the discrepancy score between felt age and chronological age (i.e., self-perceived age), and the average score was -0.10 . This value was similar to that reported by [17], who employed the same methodology to calculate self-perceived age. Therefore, the participants in both studies had similar characteristics.

We found a significant relationship between social activity and self-perceived age. This suggests that the ability to use new equipment (e.g., DVD player, mobile phone), the degree of intellectual activity (e.g., the ability to gather health-related information) and the strength of social roles (such as participation in community activities) may result in a younger self-perceived age. The above findings pertaining to the association of walking speed [7], muscular strength [3] and cognitive function [6] with subjective age indicate that these are important factors that help maintain the feeling of youthfulness among elderly people. These may also contribute to the prevention of frailty. Frailty is generally classified into "physical frailty" [32] (e.g., decrease in walking speed and muscle strength), "mental/psychological frailty" (e.g., mild cognitive impairment) and "social frailty" (e.g., impaired human relationships and decrease in social support). According to the "integral conceptual model of frailty" [33], physical, mental, psychological and social frailty interact with each other and lead to negative health outcomes. The relationship between self-perceived age and high-level functional capacity is believed to reflect the elements of mental/psychological and 
social frailty. From the above, the results of this study indicate that active participation of society and recommendation of community activities for the elderly rejuvenate their feelings and may contribute to prevention of each type of frail.

Moreover, in the subanalyses in which the elderly participants were classified into young-old and old-old subjects, a significant association was found between JST-IC score and self-perceived age in all regression models. On the contrary, among the input variables, some variables such as disease burden and self-rated health showed a significant association in the young-old or old-old subgroups. Among these variables, fall history was not a significant variable in the young-old subgroup; however, it was a significant influential factor in the old-old subgroup. An opposite phenomenon was observed with respect to the variable "fear of falling". This indicates the possibility that the effects of the fear of falling or of an actual fall may differ between the young-old and old-old. We speculate that a history of fall has a negative influence on higher living function, particularly in the old-old elderly.

An analysis based on the presence or absence of physical activity showed that the self-perceived age was significantly lower in subjects who had an established exercise habit. This result suggests that the implementation of habitual exercise has a rejuvenating effect and fosters the feeling of youthfulness.

The limitations of this study should be acknowledged. Firstly, given that this was a cross-sectional study, it was not possible to clarify the causal relationship between self-perceived age and high-levelfunctional capacity. Secondly, the information on disease burden was self-reported, and the medical diagnosis was not confirmed by a doctor. Thirdly, the survey has a low response rate; the respondents may have been limited to elderly people who are interested in health, thus possibly leading to selection bias.

The KAGUYA project is a prospective five-year study. We plan to clarify the causal relationships between self-perceived age and influential factors from various aspects by a scheduled second survey.

\section{Conclusion}

In this study, younger self-perceived age was significantly associated with the level of functional capacity, thus resulting in a higher level of functioning elderly who are independent in daily life. The same phenomenon was observed even when participants were classified into young-old and old-old subgroups. On the contrary, the influence of fear of falling and history of falls differed between the two groups; this result suggests that a history of fall has a negative influence on the functional capacity of old-old elderly. Physical activity was also related to functional capacity, and elderly people who have exercise habits had a significantly younger self-perceived age. From these results, maintaining feelings of being younger than the chronological age may promote social participation and physical activity and contribute to the prevention of frailty. 


\section{Ethics Approval and Consent to Participate}

This study was conducted with the approval of the Ethics Committee of Kio University (Approval No. H27-34). All participants provided informed consent for data collection and storage.

\section{Funding}

This study was partially supported by the Ministry of Education, Culture, Sports, Science and Technology of Japan (Private University Strategic Research Base Formation Project, No. S1591009L).

\section{Authors' Contributions}

KT, DM, JM and YN conceived and designed the experiments. KT and JM performed the experiments. KT, DM and MM analysed the data. KT wrote the paper. All authors read and approved the final manuscript.

\section{Conflicts of Interest}

The authors declare no conflicts of interest regarding the publication of this paper.

\section{References}

[1] Stephan, Y., Caudroit, J. and Chalabaev, A. (2011) Subjective Health and Memory Self-Efficacy as Mediators in the Relation between Subjective Age and Life Satisfaction among Older Adults. Aging Ment Health, 15, 428-436. https://doi.org/10.1080/13607863.2010.536138

[2] Stephan, Y., Demulier, V. and Terracciano, A. (2012) Personality, Self-Rated Health, and Subjective Age in a Life-Span Sample: The Moderating Role of Chronological Age. Psychol Aging, 27, 875-880.

https://doi.org/10.1037/a0028301

[3] Stephan, Y., Chalabaev, A., Kotter-Grühn, D. and Jaconelli, A. (2013) Feeling Younger, Being Stronger: An Experimental Study of Subjective Age and Physical Functioning among Older Adults. The Journals of Gerontology. Series B, 68, 1-7. https://doi.org/10.1093/geronb/gbs037

[4] Stephan, Y., Caudroit, J., Jaconelli, A. and Terracciano, A. (2014) Subjective Age and Cognitive Functioning: A 10-Year Prospective Study. American Journal of Geriatric Psychiatry, 22, 1180-1187. https://doi.org/10.1016/j.jagp.2013.03.007

[5] Choi, N.G., DiNitto, D.M. and Kim, J. (2014) Discrepancy between Chronological Age and Felt Age: Age Group Difference in Objective and Subjective Health as Correlates. Journal of Aging and Health, 26, 458-473.

https://doi.org/10.1177/0898264314523449

[6] Ihira, H., Furuna, T., Mizumoto, A., Makino, K., Saitoh, S., Ohnishi, H., Shimada, H. and Makizako, H. (2015) Subjective Physical and Cognitive Age among Community-Dwelling Older People Aged 75 Years and Older: Differences with Chronological Age and Its Associated Factors. Aging \& Mental Health, 19, 756-761. https://doi.org/10.1080/13607863.2014.967169

[7] Stephan, Y., Sutin, A.R. and Terracciano, A. (2015) Feeling Younger, Walking Faster: Subjective Age and Walking Speed in Older Adults. Age, 37, 86. https://doi.org/10.1007/s11357-015-9830-9 
[8] Infurna, F.J., Gerstorf, D., Robertson, S., Berg, S. and Zarit, S.H. (2010) The Nature and Cross-Domain Correlates of Subjective Age in the Oldest Old: Evidence from the OCTO Study. Psychology and Aging, 25, 470-476.

https://doi.org/10.1037/a0017979

[9] Keyes, C.L. and Westerhof, G.J. (2012) Chronological and Subjective Age Differences in Flourishing Mental Health and Major Depressive Episode. Aging and Mental Health, 16, 67-74. https://doi.org/10.1080/13607863.2011.596811

[10] Stephan, Y., Sutin, A.R. and Terracciano, A. (2015) Younger Subjective Age Is Associated with Lower C-Reactive Protein among Older Adults. Brain, Behavior, and Immunity, 43, 33-36. https://doi.org/10.1016/j.bbi.2014.07.019

[11] Rippon, I. and Steptoe, A. (2015) Feeling Old vs Being Old: Associations between Self-Perceived Age and Mortality. JAMA Internal Medicine, 175, 307-309.

https://doi.org/10.1001/jamainternmed.2014.6580

[12] Caudroit, J., Stephan, Y., Chalabaev, A. and Le Scanff, C. (2012) Subjective Age and Social-Cognitive Determinants of Physical Activity in Active Older Adults. Journal of Aging and Physical Activity, 20, 484-496.

https://doi.org/10.1123/japa.20.4.484

[13] Shrira, A., Bodner, E. and Palgi, Y. (2014) The Interactive Effect of Subjective Age and Subjective Distance-to-Death on Psychological Distress of Older Adults. Aging and Mental Health, 18, 1066-1070. https://doi.org/10.1080/13607863.2014.915925

[14] Bergland, A., Nicolaisen, M. and Thorsen, K. (2014) Predictors of Subjective Age in People Aged 40 - 79 Years: A Five-Year Follow-Up Study. The Impact of Mastery, Mental and Physical Health. Aging \& Mental Health, 18, 653-661. https://doi.org/10.1080/13607863.2013.869545

[15] Ayalon, L., Palgi, Y., Avidor, S. and Bodner, E. (2016) Accelerated Increase and Decrease in Subjective Age as a Function of Changes in Loneliness and Objective Social Indicators over a Four-Year Period: Results from the Health and Retirement Study. Aging \& Mental Health, 20, 743-751. https://doi.org/10.1080/13607863.2015.1035696

[16] Lawton, M. (1972) Research, Planning, and Action for the Elderly. In: Kent, D.P., Kastenbaum, R. and Sherwood, S., Eds., The Power and Potential of Social Science, Behavioral Publications, New York, 122-143.

[17] Stephan, Y., Sutin, A.R., Luchetti, M. and Terracciano, A. (2016) Feeling Older and the Development of Cognitive Impairment and Dementia. The Journals of Gerontology. Series B, Psychological Sciences and Social Sciences, 72, 966-973. https://doi.org/10.1093/geronb/gbw085

[18] Fujiwara, Y., Shinkai, S., Kumagai, S., Amano, H., Yoshida, Y., Yoshida, H., Kim, H., Suzuki, T., Ishizaki, T., Haga, H. and Watanabe, S. (2003) Longitudinal Changes in Higher-Level Functional Capacity of an Older Population Living in a Japanese Urban Community. Archives of Gerontology and Geriatrics, 36, 141-153. https://doi.org/10.1016/S0167-4943(02)00081-X

[19] Koyano, W., Shibata, H. and Nakazato, K. (1987) Measurement of Competence in the Elderly Living at Home: Development of an Index of Competence. Nihon Koshu Eisei Zasshi, 34, 109-115.

[20] Iwasa, H., Masui, Y., Inagaki, H., Yoshida, Y., Shimada, H., Otsuka, R., Kikuchi, K., Nonaka, K., Yoshida, H., Yoshida, H. and Suzuki, T. (2017) Assessing Competence at a Higher Level among Older Adults: Development of the Japan Science and Technology Agency Index of Competence (JST-IC). Aging Clinical and Experimen- 
tal Research, 30, 383-393. https://doi.org/10.1007/s40520-017-0786-8

[21] Koyano, W., Shibata, H., Nakazato, K., Haga, H. and Suyama, Y. (1991) Measurement of Competence: Reliability and Validity of the TMIG Index of Competence. Archives of Gerontology and Geriatrics, 13, 103-116. https://doi.org/10.1016/0167-4943(91)90053-S

[22] Iwasa, H., Masui, Y., Inagaki, H., Yoshida, Y., Shimada, H., Otsuka, R., Kikuchi, K., Nonaka, K., Yoshida, H., Yoshida, H. and Suzuki, T. (2015) Development of the Japan Science and Technology Agency Index of Competence to Assess Functional Capacity in Older Adults: Conceptual Definitions and Preliminary Items. Gerontology and Geriatric Medicine, 1.

[23] Ministry of Health, Labor and Welfare (2003) Health Japan 21 (the Second Term): National Health and Nutrition Survey.

http://www.mhlw.go.jp/seisakunitsuite/bunya/kenkou_iryou/kenkou/kenkounippo n21/en/eiyouchousa/index.html

[24] Hoyl, M.T., Alessi, C.A., Harker, J.O., Josephson, K.R., Pietruszka, F.M., Koelfgen, M., Mervis, J.R., Fitten, L.J. and Rubenstein, L.Z. (1999) Development and Testing of a Five-Item Version of the Geriatric Depression Scale. Journal of the American Geriatrics Society, 47, 873-878. https://doi.org/10.1111/j.1532-5415.1999.tb03848.x

[25] Rinaldi, P., Mecocci, P., Benedetti, C., Ercolani, S., Bregnocchi, M., Menculini, G., Catani, M., Senin, U. and Cherubini, A. (2003) Validation of the Five-Item Geriatric Depression Scale in Elderly Subjects in Three Different Settings. Journal of the American Geriatrics Society, 51, 694-698. https://doi.org/10.1034/j.1600-0579.2003.00216.x

[26] Suzuki, T. (2013) Research Institute of Science and Technology for Society: Strategic Basic Research Programs. https://ristex.jst.go.jp/pdf/korei/JST_1115090_10102752_suzuki_ER_2.pdf

[27] Hayflick, L. (2000) The Future of Ageing. Nature, 408, 267-269. https://doi.org/10.1038/35041709

[28] Fries, J. (1990) Aging Well. Adison Wesley, Merio Park.

[29] Rikli, R. and Jones, C. (1999) Functional Fitness Normative Scores for Community-Residing Older Adults, Ages 60-94. Journal of Aging and Physical Activity, 7, 162-181. https://doi.org/10.1123/japa.7.2.162

[30] Donato, A.J., Tench, K., Glueck, D.H., Seals, D.R., Eskurza, I. and Tanaka, H. (2003) Declines in Physiological Functional Capacity with Age: A Longitudinal Study in Peak Swimming Performance. Journal of Applied Physiology, 94, 764-769. https://doi.org/10.1152/japplphysiol.00438.2002

[31] Kleinspehn-Ammerlahn, A., Kotter-Grühn, D. and Smith, J. (2008) Self-Perceptions of Aging: Do Subjective Age and Satisfaction with Aging Change during Old Age? The Journals of Gerontology. Series B, Psychological Sciences and Social Sciences, 63, 377-385. https://doi.org/10.1093/geronb/63.6.P377

[32] Fried, L.P., Tangen, C.M., Walston, J., Newman, A.B., Hirsch, C., Gottdiener, J., Seeman, T., Tracy, R., Kop, W.J., Burke, G. and McBurnie, M.A. (2001) Frailty in Older Adults: Evidence for a Phenotype. The Journals of Gerontology. Series A, Biological Sciences and Medical Sciences, 56, M146-M156. https://doi.org/10.1093/gerona/56.3.M146

[33] Gobbens, R.J., Luijkx, K.G., Wijnen-Sponselee, M.T. and Schols, J.M. (2010) In Search of an Integral Conceptual Definition of Frailty: Opinions of Experts. Journal of the American Medical Directors Association, 11, 338-343.

https://doi.org/10.1016/j.jamda.2009.09.015 